\title{
Kanamycin Sulphate Loaded PLGA-Vitamin-E-TPGS Long Circulating Nanoparticles Using Combined Coating of PEG and Water-Soluble Chitosan
}

\author{
Sanaul Mustafa, V. Kusum Devi, and Roopa S. Pai \\ Pharmaceutics Division, Faculty of Pharmacy, Al-Ameen College of Pharmacy, Bangalore, Karnataka 560027, India \\ Correspondence should be addressed to V. Kusum Devi; vkusumdevi13@yahoo.in
}

Received 29 August 2016; Revised 29 November 2016; Accepted 21 December 2016; Published 2 March 2017

Academic Editor: Philippe Maincent

Copyright (C) 2017 Sanaul Mustafa et al. This is an open access article distributed under the Creative Commons Attribution License, which permits unrestricted use, distribution, and reproduction in any medium, provided the original work is properly cited.

\begin{abstract}
Kanamycin sulphate (KS) is a Mycobacterium tuberculosis protein synthesis inhibitor. Due to its intense hydrophilicity, KS is cleared from the body within $8 \mathrm{~h}$. KS has a very short plasma half-life $(2.5 \mathrm{~h})$. KS is used in high concentrations to reach the therapeutic levels in plasma, which results in serious nephrotoxicity/ototoxicity. To overcome aforementioned limitations, the current study aimed to develop KS loaded PLGA-Vitamin-E-TPGS nanoparticles (KS-PLGA-TPGS NPs), to act as an efficient carrier for controlled delivery of KS. To achieve a substantial extension in blood circulation, a combined design, affixation of polyethylene glycol (PEG) to KS-PLGA-TPGS NPs and adsorption of water-soluble chitosan (WSC) (cationic deacetylated chitin) to particle surface, was raised for surface modification of NPs. Surface modified NPs (KS-PEG-WSC NPs) were prepared to provide controlled delivery and circulate in the bloodstream for an extended period of time, thus minimizing dosing frequency. In vivo pharmacokinetics and in vivo biodistribution following intramuscular administration were investigated. NPs surface charge was close to neutral $+3.61 \mathrm{mV}$ and significantly affected by the WSC coating. KS-PEG-WSC NPs presented striking prolongation in blood circulation, reduced protein binding, and long drew-out the blood circulation half-life with resultant reduced kidney sequestration vis-à-vis KS-PLGATPGS NPs. The studies, therefore, indicate the successful formulation development of KS-PEG-WSC NPs with reduced frequency of dosing of KS indicating low incidence of nephrotoxicity/ototoxicity.
\end{abstract}

\section{Introduction}

KS is an aminoglycosides (AGs) second-line antimycobacterial agent [1]. KS is considerably a large molecule that is often used in injection form due to poor absorption through the gastrointestinal tract [2]. KS has a very short plasma halflife $(2.5 \mathrm{~h})$. In adults, KS recommended therapeutic dose is $15 \mathrm{mg} / \mathrm{kg} /$ day in equally divided intervals [3]. KS is used in high concentrations to reach the therapeutic levels in plasma and lung, which results in serious nephrotoxicity [4] and acquisition of KS resistance-TB [5], which are responsible for discontinuation of KS therapy. Due to its intense hydrophilicity, KS encounters several problems such as inadequate penetration into the cells and rapid elimination due to both efficient renal filtration and low level of association to plasma proteins [6] and belongs to Biopharmaceutics Classification System (BCS) class III. The high solubility ( $\log P$ of -3.1$)$ makes KS a possible candidate for delivery through NPs as this would not only provide sustained release but also might demonstrate a lymphatic uptake and an averting rapid renal filtration of KS [7]. This will reduce the therapeutic dose, dose dependent side effects (such as nephrotoxicity), and acquisition of KS resistance-TB related to long term use of KS [8].

Efforts are being made to advance the current drug therapy by the use of nanoparticulate systems for controlled drug delivery. Till date, inhalable spray and freeze dry KS microparticles are reported [9]. Gold NPs are reported to confirm binding affinity and interacting region of the ssDNA aptamer for KS after intrapulmonary administration [10]. Recently, the use of polymeric NPs was encouraged to provide controlled delivery, overcome rapid elimination, and reduce toxicity over the age old delivery approaches like the tablet and transdermal patch $[11,12]$.

Biodegradable polymeric PLGA NPs are of particular interest from the pharmaceutical viewpoint $[13,14]$. Chitosan 
NPs of AGs reported coupling of Chitosan NPs with dextran significantly improving encapsulation efficiency of AGs and enhancing the lung uptake of drug [15].

D- $\alpha$-tocopheryl polyethylene glycol 1000 succinate (Vitamin-E-TPGS) is a water-soluble derivative of vitamin E, which has been found to be a good emulsifier or solubilizer or absorption enhancer of high emulsification efficiency [16, 17]. Drug-loaded PLGA-TPGS emulsified NPs have shown higher encapsulation efficiency, cellular uptake, longer half-life, and elevated therapeutic effects of the formulated drug [18].

Polymeric NPs as drug carriers are rapidly removed from the blood stream through phagocytosis by the cell of the reticuloendothelial system (RES) after their intravenous administration is one of the major problems $[19,20]$. Adsorption of plasma proteins (opsonins) onto the surface of NPs is vital to the process of phagocytic recognition [19]. A commonly used method of masquerading NPs is PEGylation, the use of surface adsorbed groups which can hinder the hydrophobic and electrostatic interactions that help plasma proteins bind to NP surface and, thus, the formation of the so-called "stealth" NPs, which can escape rapid uptake by the phagocytic cells [21].

A lot of research carried out with stealth NPs focuses on intravenously administered NPs, but very modest information is known concerning the protein binding and thus in vivo pharmacokinetics and biodistribution of WSC coated NPs after intramuscular administration. Intravenous administration of liposomes and solid lipid NPs to mice caused slower renal clearance, increased half-life, and increased AUC in lung [22].

It is hypothesized that when protein binding of the NPs is decreased uptake by macrophages and tissues will be reduced [23]. Further, not much is known about the biodistribution of intramuscularly administered stealth NPs. Hydrophilic coatings (polysaccharide such as dextran) have been shown to reduce the uptake of NPs by the alveolar macrophage (AM) [15]. Particular interest has been given to chitosan due to its biocompatibility, biodegradability, and nontoxicity [24].

However, to our great knowledge, to date, this is the first comprehensive study investigating the effect of surface modified KS loaded PLGA-TPGS NPs with PEG-WSC on their pharmacokinetic, in vivo biodistribution and long circulation after intramuscular administration to rats. Thus, in the present study, we investigate the potential of KS loaded PLGA-TPGS NPs coated with PEG-WSC in augmenting sustained release of $\mathrm{KS}$ to help guard against acquisition $\mathrm{KS}$ resistance-TB and reduce toxicity.

\section{Materials and Methods}

2.1. Materials. KS was provided ex-gratis by Karnataka Antibiotic, Karnataka, India. Poly (lactic-co-glycolic acid) in a $50: 50$ molar ratio (MW 14,500 Da) and an inherent viscosity of $0.53 \mathrm{dl} / \mathrm{g}$ (Resomer RG $504 \mathrm{H}$ ) was received as gift sample from $\mathrm{M} / \mathrm{s}$ Boehringer Ingelheim Pharma $\mathrm{GmbH} \&$ Co. KG (Binger Str, Ingelheim, Germany). d- $\alpha$-Tocopheryl polyethylene glycol 1000 succinate (Vitamin-E-TPGS) was supplied ex-gratis by M/s ISOCHEM S.A, Gennevilliers,
France. Polyethylene glycol (MW 5,000 Da) and water-soluble chitosan were purchased from M/s Sigma Aldrich, Mumbai, India. The solvents such as disodium tetraborate, sodium octanesulphonate, and other chemicals employed for liquid chromatographic studies were all of HPLC grade.

\subsection{Methods}

2.2.1. Determination of KS by HPLC. The chromatographic separation was performed on a Phenomenex $\mathrm{C}_{18}$ column (250 $\mathrm{mm} \times 4.6 \mathrm{~mm}$ I.D., $5 \mathrm{~mm}$ particle size), under isocratic conditions using UV detection at $290 \mathrm{~nm}$, based on the conditions described by [25] with modifications. The mobile phase was composed of $0.1 \mathrm{M}$ disodium tetraborate $(\mathrm{pH} 9.0$ ) and water $(25: 75, \mathrm{v} / \mathrm{v})$ supplemented with $0.5 \mathrm{~g} / \mathrm{L}$ sodium octanesulphonate. The flow rate was set at $1 \mathrm{ml} / \mathrm{min}$ and the injected sample volume was $20 \mu \mathrm{l}$. The assay was linear over the KS concentration range of $120-840 \mu \mathrm{g} / \mathrm{ml}$. The limits of detection and quantification of $\mathrm{KS}$ were $60 \mu \mathrm{g} / \mathrm{mL}$ and $120 \mu \mathrm{g} / \mathrm{mL}$, respectively.

2.2.2. Preparation of KS Loaded PLGA-TPGS NPs. KS loaded PLGA-TPGS NPs were prepared in a ratio of $(1: 2)$ based on solvent emulsion-evaporation method, using Ultra Turrax IKA T25 digital high shear homogenizer [26]. In brief, $400 \mathrm{mg} \mathrm{KS}$ was dissolved in $30 \mathrm{ml}$ of aqueous phase. The resulting solution was mixed with $800 \mathrm{mg}$ of PLGA dissolved in $50 \mathrm{ml}$ of dichloromethane. The organic solution was then emulsified with $100 \mathrm{ml}$ of a TPGS $(0.25 \%)$ aqueous solution, while homogenizing at $16,000 \times \mathrm{g}$ for $30 \mathrm{~min}$. The resulting emulsion was placed on the magnetic stirrer plate and continuously stirred at room temperature to evaporate organic solution for $8 \mathrm{~h}$. The NPs were collected by centrifugation at $12,000 \times \mathrm{g}$ for $30 \mathrm{~min}$ and washed four times with distilled water. The NPs were then lyophilized and stored at $4^{\circ} \mathrm{C}$ until further analysis. Blank (without drug) NPs were also prepared using the same method.

2.2.3. Surface Modification of KS-PLGA-TPGS NPs. 2\% PEG was dissolved in the organic phase as matrix polymer and then emulsified into a TPGS $0.25 \%(\mathrm{w} / \mathrm{v})(100 \mathrm{ml})$ solution by high speed homogenizer to form NPs suspension. In this protocol, WSC was dissolved in water to form a solution and was added to the above suspension of the NPs before freeze drying; the percentage of the WSC was calculated to make the total $0.7 \%(\mathrm{w} / \mathrm{v})$ of the WSC in the final solution $(100 \mathrm{ml})$ as surface modifying agent. The suspension was then lyophilized for 2 days [23].

2.2.4. In Vitro Protein Binding Assays of KS-PEG-WSC NPs. The NPs protein binding was analyzed as method described previously for protein adsorption to polymeric NPs. Pooled rat plasma was taken and stored at $-20^{\circ} \mathrm{C}$. In brief, samples were made in different ratios of NPs suspension to plasma $(60: 40,70: 30,80: 20$, and $90: 10(\mathrm{v} / \mathrm{v}))$ to a total volume of $400 \mu \mathrm{l}$. NPs suspension/plasma was incubated for $2 \mathrm{~h}$ at room temperature and then centrifuged at $12,000 \times \mathrm{g}$ for $30 \mathrm{~min}$ to achieve a NPs pellet. The pellet was washed once with $500 \mu \mathrm{l}$ 
Mcllvaine's buffer at $\mathrm{pH} 7.5$ to eliminate any extra unbound protein [27]. Then, Bradford assay was used to determine the concentration of protein that did not bind to the NPs [28].

\subsection{Formulation Characterization}

2.3.1. Particle Size $\left(D_{n m}\right)$ and Zeta $(\zeta)$ Potential Measurements. The particle size of KS-PEG-WSC NPs and KS-PLGA-TPGS NPs was determined using Malvern Zetasizer Nano S90 (Malvern Instruments Ltd., U.K) and the zeta potential was measured using Laser Doppler Velocimetry (LDV) with a Malvern Zetasizer Nano ZS (Malvern Instruments Ltd., U.K). Samples were diluted in MilliQ ${ }^{\mathrm{TM}}$ water before measurement.

2.3.2. Transmission Electron Microscopy. The morphology of the KS-PEG-WSC NPs was observed using transmission electron microscope (TEM) attached with a mega view II digital camera (H 7500, Hitachi, Tokyo, Japan). A drop of sample diluted with water was placed on a copper grid and the excess was drawn off with a filter paper. Samples were subsequently stained with $2 \%$ of uranyl acetate solution for $30 \mathrm{~s}$. The image was magnified and focused on a layer of photographic film.

2.3.3. Drug Encapsulation Efficiency $\left(D_{E E}\right)$. The $D_{\mathrm{EE}}$ was determined by the separation of KS-PEG-WSC NPs and KS-PLGA-TPGS NPs from the aqueous medium containing nonassociated KS by ultracentrifugation (REMI high speed, cooling centrifuge, REMI Corporation, India) at 15,000 $\times \mathrm{g}$ for $30 \mathrm{~min}$, at $4^{\circ} \mathrm{C}$. The unencapsulated $\mathrm{KS}$ was determined using HPLC.

2.3.4. In Vitro Drug Release Studies. Release studies were carried out for KS-PEG-WSC NPs and KS-PLGA-TPGS NPs. Drug-loaded NPs corresponding to $500 \mathrm{mg}$ of drug were placed in dialysis cellulose membrane bags (cellophane membrane, molecular weight cut-off 10,000-12,000, Hi-Media, India). The bags were sealed closely by clamps after $1 \mathrm{ml}$ of Phosphate Buffer Solution (PBS) was dropped into each bag. The dissolution medium consisted of a PBS (0.1 M, pH 7.4). The stirring rate was kept constant at $50 \mathrm{rpm}$, as was the temperature at $37^{\circ} \mathrm{C}$ with continuous magnetic stirring. At selected time intervals, aliquots were withdrawn from the release medium and replaced with the same amount of fresh PBS and concentrations of the released drug were determined by HPLC method.

2.3.5. Pharmacokinetic Studies. All the animal investigations were performed as per the requisite protocol approved by the Institutional Animal Ethics Committee [Letter no AACP/IAEC/Jun-2014-01]. The Committee is duly approved for the purpose of control and supervision of experiments on the animals by the Government of India. The pharmacokinetic study involved two groups. Six rats (male Wistar, weighing $250-300 \mathrm{~g}$ ) were randomly distributed amongst each group.

Group I received KS-PLGA-TPGS NPs formulation redispersed in $1 \mathrm{ml}$ of water.
Group II received KS-PEG-WSC NPs formulation redispersed in $1 \mathrm{ml}$ of water.

All the animal groups received a dose equivalent to $15 \mathrm{mg}$ of KS per $\mathrm{kg}$ of body weight [3]. After intramuscular drug administration, the rats kept in cages were allowed access to food and water ad libitum. Serial aliquots of the blood samples $(100 \mu \mathrm{l}$ each) were withdrawn from the retroorbital plexus under mild ether anesthesia at $0,0.5,1,3,6,12$, and every $24 \mathrm{~h}$ for 6 days in the heparinized microcentrifuge tubes (50 units heparin/ml of blood). Plasma was harvested by centrifugation at $15,000 \times \mathrm{g}$ for $15 \mathrm{~min}$ and stored at $-20^{\circ} \mathrm{C}$ until analyzed. Acetonitrile was added to precipitate the plasma proteins. Thereafter, samples were vortexed and centrifuged at $15,000 \times \mathrm{g}$ for $20 \mathrm{~min}$ and were analyzed by HPLC. The competence of nanoparticulate formulation was assessed by administering pure drug intramuscularly and measuring the blood levels at $0,0.5,1,2,3,6,12$, and $24 \mathrm{~h}$. Noncompartmental pharmacokinetic parameters for extravascular input, that is, $C_{\max }, T_{\max }, \mathrm{AUC}_{0-\infty}, T_{1 / 2}, K_{e}$, and MRT, were computed by choosing Kinetica 5.0.11 version software (Thermo Fisher Scientific Inc. Waltham, USA).

2.3.6. In Vivo Biodistribution Assay. The animals and dosing protocol were the same as in pharmacokinetic study. To study in vivo biodistribution, 18 rats were randomly divided into two groups of 9 rats. Group I was treated with KS-PLGATPGS NPs and Group II was treated with KS-PEG-WSC NPs. Intramuscular administration was performed on the same day and the three rats of each group were sacrificed by cervical dislocation under general pentobarbital anesthesia on Day 1 , Day 5, and Day 7. Heart, lungs, kidneys, and spleen as well as plasma were collected and processed immediately for analysis by HPLC $[22,25]$.

2.3.7. Statistical Analysis. One-way ANOVA was used to compare the data. The differences in the means were significant and post hoc pair wise comparisons were conducted using Newman-Keuls multiple comparison using GraphPad Prism software ver 5.0 (M/s GraphPad Software Inc., California, USA). For in vivo biodistribution analysis was confirmed by Bonferroni's as a post hoc test and the one-way ANOVA was used to compare the data using GraphPad Prism software ver 5.0 (M/s GraphPad Software Inc., California, USA) [26].

2.3.8. Stability Studies. KS-PLGA-TPGS NPs and KS-PEGWSC NPs were subjected to stability studies, carried out at $25 \pm 2^{\circ} \mathrm{C} / 60 \% \pm 5 \% \mathrm{RH}$, as per the ICH guidelines for the climatic zone IV. The formulation was assayed periodically, at the time points of $0,1,3$, and 6 months, for particle size, drug encapsulation efficiency, and zeta potential [26].

\section{Results}

3.1. Particle Size and Drug Encapsulation Efficiency. The solvent emulsion-evaporation method was successfully employed to fabricate NPs for intramuscular drug delivery. The yield of the polymeric NPs was $94.37 \%$ with this protocol. 


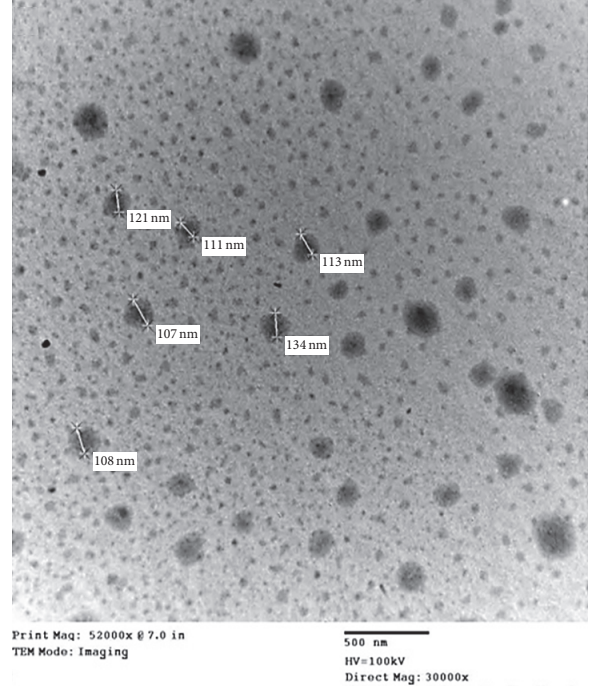

(a)

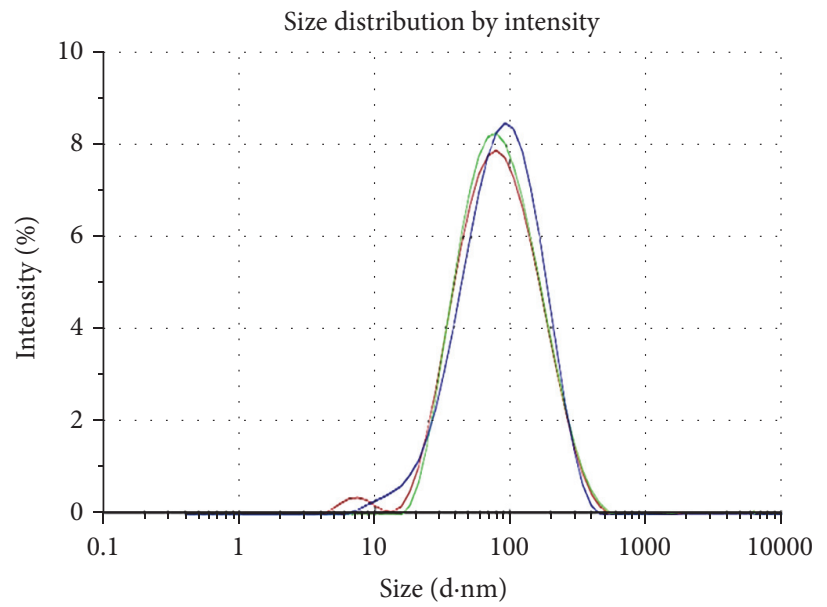

(b)

FIGURE 1: (a) TEM image shows particle size of KS-PEG-WSC NPs formulation. (b) Size distribution of KS-PEG-WSC NPs formulation. KS-PEG-WSC NPs: Kanamycin-polyethylene glycol-water-soluble chitosan-nanoparticles; TEM: transmission electron microscopy.

The KS-PLGA-TPGS NPs formed were uniform and discrete and of average particle size of $\sim 86.71 \mathrm{~nm}$ with a polydispersity index less than 0.267. Postinclusion of PEG-WSC made only a $25 \mathrm{~nm}$ raise of the particle size $(113.16 \mathrm{~nm})$. KS encapsulation efficiency in PLGA-TPGS NPs and PEG-WSC NPs was found to be $66.31 \pm 4.12 \%$ and $74.45 \pm 2.83 \%$, respectively. The presence of PEG-WSC modifier significantly improved the drug encapsulation efficiency; the reason is that the drug was entrapped within the PEG and WSC chains or this may be the effect of emulsification outcome of the PLGA-TPGS [13].

3.2. Transmission Electron Microscopy. The TEM image (Figure 1(a)) clearly reveals that most of the emulsion particles of KS-PEG-WSC NPs formulation were below $200 \mathrm{~nm}$ in size and were spherical in shape. Figure 1(b) illustrates the Dnm distribution and markedly reveals the globule size of the OPT formulation as $113.16 \mathrm{~nm}$.

3.3. In Vitro Protein Binding of KS-PLGA-TPGS NPs and KSPEG-WSC NPs. Different ratios of NPs: plasma were taken to evaluate the Vroman effect. This relates to a possible adsorption of proteins on the surface sites of NPs [29]. At a $60: 40,70: 30,80: 20$, and $90: 10$ plasma volume, KSPLGA-TPGS NPs showed an average protein binding of 19.72 $\pm 6.19 \%, 27.62 \pm 3.45 \%, 31.64 \pm 4.81$, and $39.12 \pm 4.24 \%$, respectively. A similarity between this formulation and a similar formulation coated with PEG- (2\%) WSC (0.7\%) illustrated highly momentous variation in plasma protein binding $16.41 \pm 3.45\left({ }^{* * *} p<0.001\right)$ at a plasma volume of $40 \%$. The improved protein binding for KS-PEG-WSC NPs noticed at $30 \%(18.73 \pm 3.26 \%), 20 \%(21.74 \pm 4.21 \%)$, and $10 \%$ $(29.44 \pm 2.76 \%)$ plasma volume was an unpredicted result since surface modification with WSC is well documented to

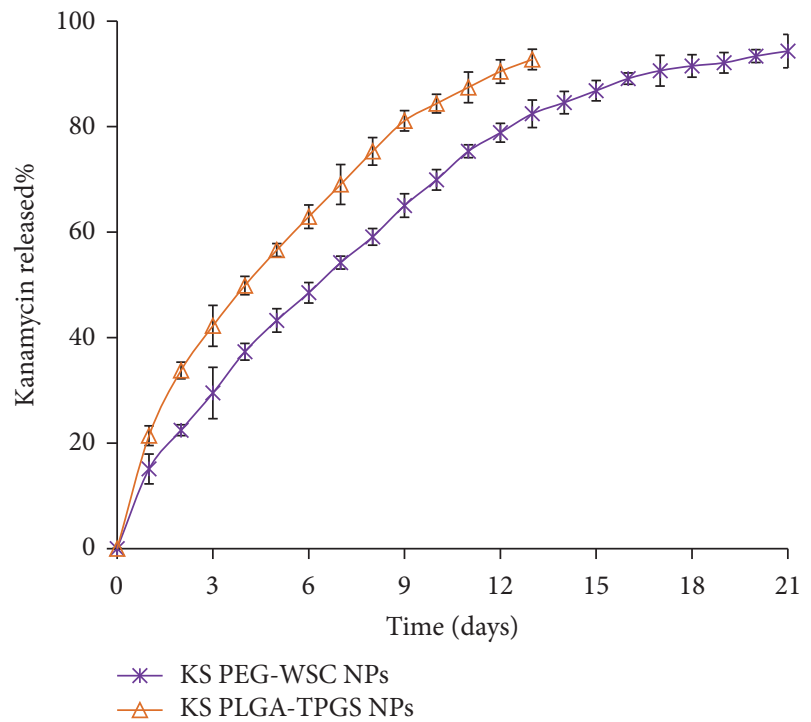

Figure 2: In vitro release profile of KS-PEG-WSC NPs and KSPLGA-TPGS NPs in $\mathrm{pH} 7.4 \mathrm{PBS}$. Data represented the mean \pm SD, $n=3$.

decrease protein adsorption [30]. Statistically, no significant difference was observed between the three percentages $(30 \%$, $20 \%$, and $10 \%)\left({ }^{*} p>0.01\right)$.

3.4. In Vitro Drug Release Studies. Figure 2 presents the release profiles of KS from the PLGA-TPGS NPs and PEGWSC NPs. KS-PLGA-TPGS NPs tended to release minuscule amount of drug $\sim 21.42 \%$ within initial $24 \mathrm{~h}$, with relatively $91.56 \%$ of drug being released within 14 days, whereas KSPEG-WSC NPs showed $\sim 15.11 \%$ amount of drug release 


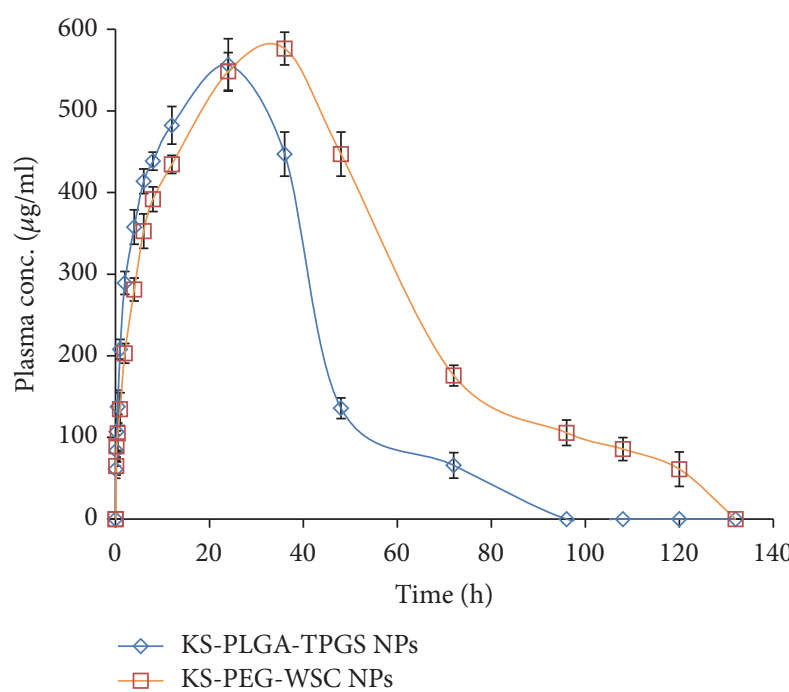

(a)

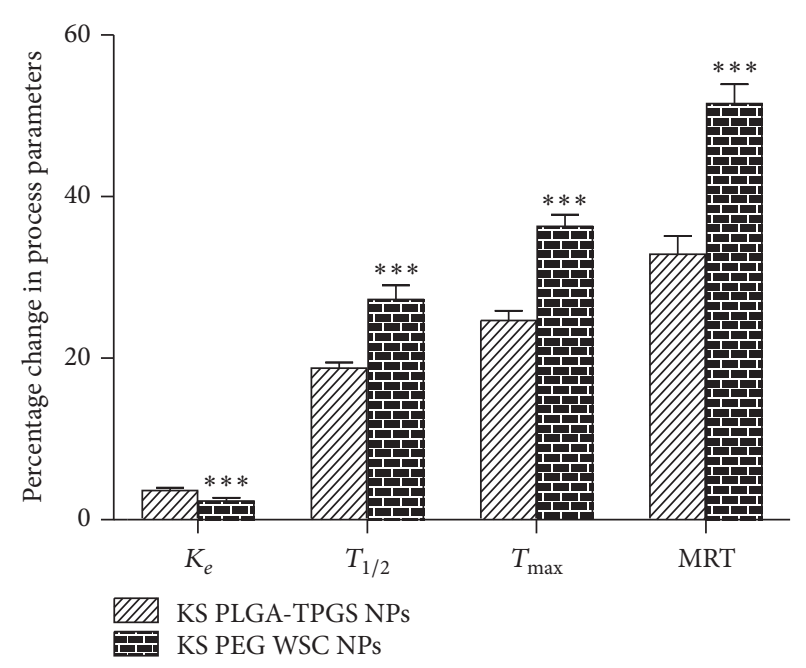

(b)

FIGURE 3: (a) Plasma drug level profiles of KS-PEG-WSC NPs and KS-PLGA-TPGS NPs. Each point represents mean of six replicates and each crossbar indicates 1 SEM and (b) change in pharmacokinetic parameters of KS-PEG-WSC NPs relative to KS-PLGA-TPGS NPs.

within initial $24 \mathrm{~h}$, followed by a slow and sustained release (93.26\%) till 21 days.

3.5. In Vivo Pharmacokinetic Assay. KS-PEG-WSC NPs showed prolonged residence in the blood circulation vis-à-vis KS-PLGA-TPGS NPs. The mean plasma concentration-time profiles (Figure 3) portray considerable higher plasma levels from KS-PEG-WSC NPs $\left({ }^{* * *} p<0.001\right)$ formulation with respect to KS-PLGA-TPGS NPs. KS-PEG-WSC NPs remained in the blood for an extended period of time (halflife, $t_{1 / 2}: 27.76 \mathrm{~h}$ ) vis-à-vis KS-PLGA-TPGS NPs (half-life, $t_{1 / 2}: 18.23 \mathrm{~h}$ ). The absorption rate of KS-PEG-WSC NPs was markedly enhanced, as observed by KS discrete improvement in various pharmacokinetic parameters, that is, $T_{\max }, T_{1 / 2}$, $C_{\max }$, and $\mathrm{AUC}_{0-\infty}$. Subsequent one-way ANOVA carried out on various pharmacokinetic parameters, namely, $T_{1 / 2}, K_{e}$, $\mathrm{AUC}_{0-\infty}$, and $T_{\max }$, implied highly statistically significant variation $\left({ }^{* * *} p<0.001\right)$ in the drug absorption potential of KS from PEG-WSC NPs. As is evident from Figures 3(a) and 3(b), the availability of KS in case of PEG-WSC NPs was almost 1.52 -fold as compared to PLGA-TPGS NPs up to 6 days, suggesting a longer circulation time of KS in the blood.

3.6. In Vivo Biodistribution Assay. The in vivo biodistribution of the NPs was greatly influenced by PEG-WSC content. KSPLGA-TPGS NPs without coating were detected in lungs, kidneys, heart, and the spleen over a period of 7 days (Figure 4). However, at the end of Day 1, faster elimination of marketed product was observed in all the organs vis-à-vis KS-PLGA-TPGS NPs and KS-PEG-WSC NPs. Minuscule amount of concentrations was detected in the plasma over the same period. It was observed that lung accumulated major portion of the administered KS-PLGA-TPGS NPs but lower lung concentration in comparison to KS-PEG-WSC NPs. The accumulation and clearance of the NPs in different organs varied. Besides the blood, the KS-PLGA-TPGS NPs were mainly found in all the heart, kidney, and spleen.

The cumulative amounts of KS PEG-WSC NPs dramatically reduced in kidney, changed little in heart and lung, and were exhibited slightly higher in spleen. The accumulation rate of KS-PEG-WSC NPs in kidney was 3.64-fold lower than that of KS-PLGA-TPGS NPs over a period of 7 days (Figure 4). The minuscule amount of concentrations in organs especially kidney indicated slower filtration of KSPEG-WSC NPs by and extended residence time in the blood.

3.7. Stability Studies. KS-PLGA-TPGS NPs and KS-PEGWSC NPs show very small variation in the formulation parameters during 6 months of storage period (Table 1).

\section{Discussion}

KS loaded PLGA-TPGS NPs surface modified with PEG and WSC was prepared in our laboratory to achieve a prolongation in systemic circulation and increased uptake in lungs (site of action). Coating was applied to prepare long circulating NPs with biodegradable polymer PLGA working as the core for drug reservoir and the water-soluble PEG and WSC accommodative as the corona designing towards the outer aqueous environment. PEG has been widely employed in drug delivery to augment the circulation time of NPs in blood. Perhaps due to electrostatic interactions between the positively charged WSC and the negatively charged PLGA, WSC coating was formed of the NPs. In addition, due to its amphiphilic nature, WSC was prone to form monolayer arrangement at the surface of NPs [31].

To our best knowledge, there has been hardly ever report on the steadiness of attaching WSC on the surface of KS loaded PLGA-TPGS NPs. A potential explanation for the positive role of the WSC coating at improving particle 

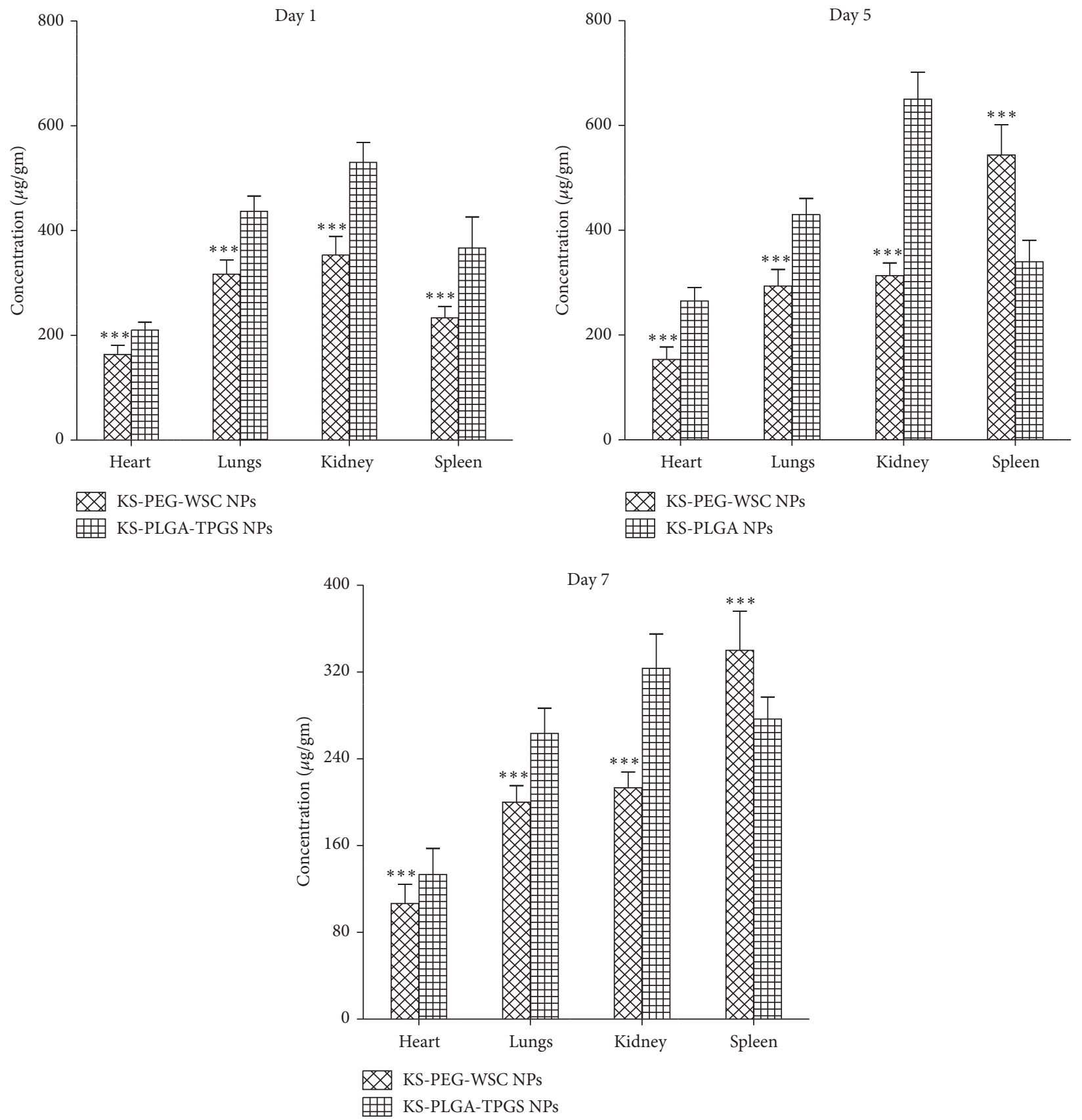

FIGURE 4: Body distribution of KS-PEG-WSC NPs and KS-PLGA-TPGS NPs after intramuscular administration to rats. Concentration of KS ( $y$-axis) was determined in different organs on Day 1, Day 5, and Day 7 after intramuscular administration.

TABLE 1: Various parameters of the KS-PLGA-TPGS NPs and KS-PEG-WSC NPs analyzed at different time points during stability studies.

\begin{tabular}{|c|c|c|c|c|c|c|}
\hline \multirow[b]{2}{*}{ Time (month) } & \multicolumn{2}{|c|}{$D_{\mathrm{nm}}$} & \multicolumn{2}{|c|}{$D_{\mathrm{EE}}(\%)$} & \multicolumn{2}{|c|}{$\zeta$ potential } \\
\hline & $\begin{array}{c}\text { KS-PLGA-TPGS } \\
\text { NPs }\end{array}$ & $\begin{array}{c}\text { KS-PEG-WSC } \\
\text { NPs }\end{array}$ & $\begin{array}{c}\text { KS-PLGA-TPGS } \\
\text { NPs }\end{array}$ & $\begin{array}{c}\text { KS-PEG-WSC } \\
\text { NPs }\end{array}$ & $\begin{array}{c}\text { KS-PLGA-TPGS } \\
\text { NPs }\end{array}$ & $\begin{array}{c}\text { KS-PEG-WSC } \\
\text { NPs }\end{array}$ \\
\hline 0 & 86.71 & 113.16 & 66.31 & 74.45 & -27.91 & +3.61 \\
\hline 1 & 86.19 & 113.03 & 65.85 & 74.21 & -27.67 & +3.53 \\
\hline 3 & 85.72 & 112.86 & 65.51 & 73.83 & -27.32 & +3.02 \\
\hline 6 & 85.36 & 112.45 & 65.22 & 73.26 & -27.13 & +2.98 \\
\hline
\end{tabular}


stability was based on an efficient steric repulsion effect produced by the hydrophilic chains of the macromolecular WSC projecting towards the external phase, which inhibited coalescence or flocculation.

This study however focused on the effect of PEG and WSC on the in vitro protein binding as well as the pharmacokinetics and in vivo biodistribution of KS-PLGA-TPGS NPs after intramuscular administration. In the present study, the augment in particle size, improvement of surface hydrophilicity, and reversal of surface charge were attained by successful "insertion" of PEG or the outer WSC layer of the NPs.

Increase in the particle size of the NPs was observed by using PEG $(5,000 \mathrm{Da}, 2 \%)$ and WSC $(0.7 \%)$ in KSPLGA-TPGS NPs, which might be owing to the multilayer depositions of PEG-WSC. This enhance in particle size was a first sign of the attachment of WSC to the surface of the NPs. The uptake of the NPs through AM decreases with increasing the particle size reaching a cut-off at around $200 \mathrm{~nm}$ [32]. The inclusion of positively charged WSC has been suggested in earlier reports to enhance uptake through AM $[33,34]$. It has also been reported that particle size less than $200 \mathrm{~nm}$ generally remains undetectable to the reticuloendothelial system (RES) and keeps on circulating over an extended period of time [35].

KS-PLGA-TPGS NPs had a negative charge of $-27.91 \mathrm{mV}$, which was reversed to positive value $(+3.61 \mathrm{mV})$ after insertion of PEG-WSC. Zeta potential for the KS-PEG-WSC NPs was lower than the KS-PLGA-TPGS NPs $\left({ }^{* *} p<0.001\right)$; it may be because of a higher concentration of PEG and inclusion of positively charged WSC adsorbed on the surface of NPs with the development of a denser surfactant coating on the NPs surface, thus, eliciting a reduced electrophoretic mobility, and an elevated absolute potential value ensures a high-energy barrier that stabilizes the nanosuspension [36]. NPs charge also influences their circulation time and clearance. However, the neutral NPs are anticipated to remain in circulation over a prolonged period of time as opsonization processes are not uniformly activated (as for charged NPs) for their elimination from the body [35].

PLGA-Vitamin-E-TPGS NPs have been reported to give high encapsulation efficiency for water-soluble drugs. Present investigation also reveals that solvent emulsion-evaporation method can lead to NPs with high encapsulation with watersoluble drug such as KS. The findings are in good agreement with literature reports [37].

In vitro protein binding assay was assessed to predict the phagocytosis of NPs by the cells of MPS. Phagocytic uptake of NPs by cells of MPS is responsible for the loss of NPs in the systemic circulation. A relationship between surface charge and opsonization was showed in vitro that neutrally charged NPs have a much lesser opsonization rate than charged NPs. Their lack of binding with opsonins would be responsible for their prolonged residence in the blood circulation. On the contrary, KS-PLGA-TPGS NPs with a higher negative zeta potential would possess a stronger affinity for plasma proteins, accounting for its relatively higher protein binding. It is indicated that when PLGA-TPGS NPs made long circulating, the protein binding differs depending on the polymeric surfactant used. The diminution of protein binding might be ascribed to the higher surface coverage which is achieved as a result of the conformation of the PEG-WSC chains in a "brush-like" configuration [38]. Thus, the PEG in combination with WSC was supposed to assume a dense brush-like conformation and act to increase the opsonins resistant properties. Their lack of binding with opsonins and then escaping phagocytosis would be likely responsible for their prolonged persistence in the blood circulation.

A sustained release pattern is a key issue in the development of colloidal drug delivery systems used in the field of nanomedicine. The in vitro release behavior of the KS-PLGATPGS NPs and KS-PEG-WSC NPs is shown in Figure 2. The initial burst release was prominent for KS-PLGA-TPGS NPs and KS-PEG-WSC NPs during the first day of release, being greater than $10-15 \%$. This effect can be because of the drug adsorbed on the surface of NPs [39]. Besides, we believe that burst release may be accounted for the passage of very small NPs which may have passed liberally across the dialysis membrane $(\sim 10-20 \mathrm{~nm})$. The initial phase of drug release however can be used fruitfully to provide a loading dose wherein $\sim 25 \%$ of the total KS present in the formulation is released within $24 \mathrm{~h}$ of its administration, while the remaining amount may help to maintain the plasma concentrations for $>24 \mathrm{~h}$. Release of the encapsulated KS from PLGA matrix was found to occur through diffusion-cum degradation-mediated process [40]. During the early phases, release occurs mostly through diffusion in the polymer matrix, while during the later phases release is mediated through both diffusion of the KS and degradation of the polymer matrix itself. It is apparent that both the emulsifier and the particle material composition determine the in vitro release behavior. Faster drug release of KS-PLGA-TPGS NPs may be attributed to the lower molecular weight of PLGA and the higher hydrophilicity of TPGS copolymer in comparison with the KS-PEG-WSC NPs.

In the current work, KS-PEG-WSC NPs were prepared and administered intramuscularly to Wistar rats that contained $\sim 16.21 \%$ free KS $(74.51 \pm 1.84 \%$, encapsulation efficiency) and significant percentage of particles were of a very small size $(113.16 \mathrm{~nm})$ such that they can be transported via lymphatic transport. The neutrally charged KS-PEG-WSC NPs do not activate any system for their elimination from the body such that they are projected to bypass AM pickup resulting in extended circulation times and since lung is the major site of action of KS, AM avoidance of KS-PEG-WSC NPs is especially expected to reduce its elimination from the body. High plasma concentration complemented with overcoming of AM uptake will present higher amounts of KS in the systemic circulation. The higher $T_{\max }$ levels coupled with higher values of $T_{1 / 2}$ and $\mathrm{AUC}_{0-\infty}$ observed in those KS-PEG-WSC NPs indisputably vouch distinct improvement in rate and extent of drug bioavailability. This augmentation in bioavailability would finally result in an increase in the intensity of therapeutic effect of KS.

A significantly $\left({ }^{* * *} p \leq 0.001\right)$ lower concentration of KS in kidney when administered in the form of NPs indicates a lower elimination of KS. This coupled with the bypass of rapid elimination of KS due to its entrapment within PEGWSC NPs can suitably explain a significant decrease in $K_{e}$ of KS $\left({ }^{* * *} p \leq 0.001\right)$ when administered as KS NPs. This effect 
may be attributed to the use of larger molecular weight PEG polymer that led to longer blood circulation half-lives for the particles in vivo [18].

The in vivo biodistribution of the NPs influenced by surface PEG-WSC content in different organs varied. The concentrations of KS-PEG-WSC NPs compared with KSPLGA-TPGS NPs significantly reduced in kidney and other organs. The major pathway for the exclusion of NPs from blood appeared to be the NPs detention in MPS organs. Typically, once polymeric NPs are opsonized and separated from the bloodstream, they are sequestered in one of the MPS organs. In the case of NPs lack of stealth properties, accumulation in the MPS organs is very fast within a minute and generally concentrates in the kidney and spleen [16].

The significant reduction in kidney for KS-PEG-WSC NPs may be due to the hidden surface created by PEG-WSC coating, with neutral-charge and brush-like conformation, which could decrease kidney filtration. A small high spleen uptake was considered to be a result of prolonged systemic circulation of KS-PEG-WSC NPs [30]. Though, we agree that PLGA-TPGS NPs do show a significant prolonged circulation, nevertheless the effect was much more prominent in case of a PEG-WSC coating at all time point studies. Thus, an advantage of PEG-WSC coating on the NPs for circumventing AM uptake was obvious. A relatively high accumulation of NPs in spleen was observed compared with KS-PLGA-TPGS NPs and extended residence time in the blood might indicate slower kidney filtration.

NPs were detected in heart, lung, kidney, and spleen over the 5 days and the plasma concentrations of KS-PEGWSC NPs were higher than that of KS-PLGA-TPGS NPs, demonstrating that the extended residence time can be obtained with intramuscular formulations. This effect may be due to surface heterogeneity and hydrophobic nature of PLGA which could further explain the presence of NPs in the spleen (representing particles that are taken up by the $M$ cells of the Peyer's patches via opsonization) and the kidney (representing particles size $>10 \mathrm{~nm}$ averting rapid kidney filtration).

The KS-PEG-WSC NPs resulted in reduced protein binding when administered intramuscularly and there is a significant increase in the percentage detected in plasma; the distribution to various organs is statistically significantly $\left({ }^{* * *} p<0.001\right)$ different from the KS-PLGA-TPGS NPs. Furthermore, these results designate that for NPs in vitro observations cannot represent or be correlated to the in vivo behavior of the NPs.

\section{Conclusions}

In the present study, PLGA-TPGS NPs bearing a combined coating of PEG and WSC were prepared. Surface charge and surface hydrophilicity were significantly affected by the nature of the coating. Combination of PEG and WSC presented an effective shielding character to the NPs as well as neutral surface charge. Also, entrapping KS into PEGWSC NPs could result in improved availability of KS in lung (sites of action), or minimum concentration in kidney (sites of rapid clearance as well as toxicity). Based on the above results, these NPs might be adequate for good long circulating efficacy and will be promising scenario as efficiently targeted drug delivery systems.

\section{Competing Interests}

The authors disclose no conflict of interests.

\section{Acknowledgments}

The authors would like to thank Dr. Shobha Rani R. H, Principal of Al-Ameen College of Pharmacy for her advice and support to carry out this research work. The authors are very grateful to Karnataka Antibiotics, Bangalore, India, for providing the gift sample of Kanamycin. They are also grateful to M/s Boehringer Ingelheim Pharma GmbH \& Co. KG (Binger Street, Ingelheim, Germany) for providing the gift sample of PLGA.

\section{References}

[1] S. Mustafa, R. S. Pai, G. Singh, and V. K. Devi, "Nanocarrierbased interventions for the management of MDR/XDR-TB," Journal of Drug Targeting, vol. 23, no. 4, pp. 287-304, 2015.

[2] R. S. Edson and C. L. Terrell, "The aminoglycosides," Mayo Clinic Proceedings, vol. 74, no. 5, pp. 519-528, 1999.

[3] J. T. Doluisio, L. W. Dittert, and J. C. LaPiana, "Pharmacokinetics of kanamycin following intramuscular administration," Journal of Pharmacokinetics and Biopharmaceutics, vol. 1, no. 3, pp. 253-265, 1973.

[4] S. Yu, Q. Wei, B. Du et al., "Label-free immunosensor for the detection of kanamycin using $\mathrm{Ag} @ \mathrm{Fe}_{3} \mathrm{O}_{4}$ nanoparticles and thionine mixed graphene sheet," Biosensors and Bioelectronics, vol. 48, pp. 224-229, 2013.

[5] A. Jain, P. Dixit, and R. Prasad, "Pre-XDR \& XDR in MDR and Ofloxacin and Kanamycin resistance in non-MDR Mycobacterium tuberculosis isolates," Tuberculosis, vol. 92, no. 5, pp. 404-406, 2012.

[6] J. A. Dijkstra, R. Van Altena, O. W. Akkerman et al., "Limited sampling strategies for therapeutic drug monitoring of amikacin and kanamycin in patients with multidrug-resistant tuberculosis," International Journal of Antimicrobial Agents, vol. 46, no. 3, pp. 332-337, 2015.

[7] L. Z. Benet, F. Broccatelli, and T. I. Oprea, "BDDCS applied to over 900 drugs," AAPS Journal, vol. 13, no. 4, pp. 519-547, 2011.

[8] R. De Jager and R. Van Altena, "Hearing loss and nephrotoxicity in long-term aminoglycoside treatment in patients with tuberculosis," International Journal of Tuberculosis and Lung Disease, vol. 6, no. 7, pp. 622-627, 2002.

[9] J. R. Manion, S. P. Cape, D. H. McAdams, L. G. Rebits, S. Evans, and R. E. Sievers, "Inhalable antibiotics manufactured through use of near-critical or supercritical fluids," Aerosol Science and Technology, vol. 46, no. 4, pp. 403-410, 2012.

[10] K.-M. Song, M. Cho, H. Jo et al., "Gold nanoparticle-based colorimetric detection of kanamycin using a DNA aptamer," Analytical Biochemistry, vol. 415, no. 2, pp. 175-181, 2011.

[11] S. Saraya, J. Kanta, N. Sarisuta et al., "Development of guava extract chewable tablets for anticariogenic activity against 
Streptococcus mutans," Journal of Pharmaceutical Sciences, vol. 35, no. 2, pp. 18-23, 2008.

[12] M. López-Cervantes, J. J. Escobar-Chávez, N. Casas-Alancaster, D. Quintanar-Guerrero, and A. Ganem-Quintanar, "Development and characterization of a transdermal patch and an emulgel containing kanamycin intended to be used in the treatment of mycetoma caused by Actinomadura madurae," Drug Development and Industrial Pharmacy, vol. 35, no. 12, pp. 1511-1521, 2009.

[13] H. Chen, Y. Zheng, G. Tian et al., "Oral delivery of DMABmodified docetaxel-loaded PLGA-TPGS nanoparticles for cancer chemotherapy," Nanoscale Research Letters, vol. 6, no. 1, pp. 1-10, 2011.

[14] G. Singh and R. S. Pai, "Pharmacokinetics and in vivo biodistribution of optimized PLGA nanoparticulate drug delivery system for controlled release of emtricitabine," Drug Delivery, vol. 21, no. 8, pp. 627-635, 2014.

[15] E. Lu, S. Franzblau, H. Onyuksel, and C. Popescu, "Preparation of aminoglycoside-loaded chitosan nanoparticles using dextran sulphate as a counterion," Journal of Microencapsulation, vol. 26, no. 4, pp. 346-354, 2009.

[16] L. Mu and S. S. Feng, "Vitamin E TPGS used as emulsifier in the solvent evaporation/extraction technique for fabrication of polymeric nanospheres for controlled release of paclitaxel (Taxol $\left.{ }^{\circledR}\right), "$ Journal of Controlled Release, vol. 80, no. 1-3, pp. 129144, 2002.

[17] L. Mu and S. S. Feng, "A novel controlled release formulation for the anticancer drug paclitaxel (Taxol $\left.{ }^{\circledR}\right)$ : PLGA nanoparticles containing vitamin E TPGS," Journal of Controlled Release, vol. 86, no. 1, pp. 33-48, 2003.

[18] Z. Zhang, S. H. Lee, and S.-S. Feng, "Folate-decorated poly(lactide-co-glycolide)-vitamin E TPGS nanoparticles for targeted drug delivery," Biomaterials, vol. 28, no. 10, pp. 18891899, 2007.

[19] D. E. Owens III and N. A. Peppas, "Opsonization, biodistribution, and pharmacokinetics of polymeric nanoparticles," International Journal of Pharmaceutics, vol. 307, no. 1, pp. 93102, 2006.

[20] A. A. Vassiliou, S. A. Papadimitriou, D. N. Bikiaris, G. Mattheolabakis, and K. Avgoustakis, "Facile synthesis of polyester-PEG triblock copolymers and preparation of amphiphilic nanoparticles as drug carriers," Journal of Controlled Release, vol. 148, no. 3, pp. 388-395, 2010.

[21] S. E. Dunn, A. G. A. Coombes, M. C. Garnett, S. S. Davis, M. C. Davies, and L. Illum, "In vitro cell interaction and in vivo biodistribution of poly(lactide-co-glycolide) nanospheres surface modified by poloxamer and poloxamine copolymers," Journal of Controlled Release, vol. 44, no. 1, pp. 65-76, 1997.

[22] J. Varshosaz, S. Ghaffari, S. F. Mirshojaei et al., "Biodistribution of amikacin solid lipid nanoparticles after pulmonary delivery," BioMed Research International, vol. 2013, Article ID 136859, 8 pages, 2013.

[23] F. Esmaeili, M. H. Ghahremani, B. Esmaeili, M. R. Khoshayand, F. Atyabi, and R. Dinarvand, "PLGA nanoparticles of different surface properties: preparation and evaluation of their body distribution," International Journal of Pharmaceutics, vol. 349, no. 2, pp. 249-255, 2008.

[24] L. Illum, "Chitosan and its use as a pharmaceutical excipient," Pharmaceutical Research, vol. 15, no. 9, pp. 1326-1331, 1998.
[25] B. Blanchaert, E. Poderós Jorge, P. Jankovics, E. Adams, and A. Van Schepdael, "Assay of kanamycin A by HPLC with direct UV detection," Chromatographia, vol. 76, no. 21, pp. 1505-1512, 2013.

[26] G. Singh and R. S. Pai, "Optimized PLGA nanoparticle platform for orally dosed trans-resveratrol with enhanced bioavailability potential," Expert Opinion on Drug Delivery, vol. 11, no. 5, pp. 647-659, 2014.

[27] B. Semete, L. Booysen, L. Kalombo, B. Ramalapa, R. Hayeshi, and H.S. Swai, "Effects of protein binding on the biodistribution of PEGylated PLGA nanoparticles post oral administration," International Journal of Pharmaceutics, vol. 424, no. 1-2, pp. 115120, 2012.

[28] X. Qian, H. Dong, X. Hu et al., "Analysis of the interferences in quantitation of a site-specifically PEGylated exendin-4 analog by the Bradford method," Analytical Biochemistry, vol. 465, pp. 50-52, 2014.

[29] S. M. Moghimi and J. Szebeni, "Stealth liposomes and long circulating nanoparticles: critical issues in pharmacokinetics, opsonization and protein-binding properties," Progress in Lipid Research, vol. 42, no. 6, pp. 463-478, 2003.

[30] Y. Sheng, C. Liu, Y. Yuan et al., "Long-circulating polymeric nanoparticles bearing a combinatorial coating of PEG and water-soluble chitosan," Biomaterials, vol. 30, no. 12, pp. 2340 2348, 2009.

[31] J. Wang, C. Liu, and P. Chi, "Aggregate formation and surface activity of partially deacetylated water-soluble chitin," Research on Chemical Intermediates, vol. 34, no. 2-3, pp. 169-179, 2008.

[32] D. A. Norris, N. Puri, and P. J. Sinko, "The effect of physical barriers and properties on the oral absorption of particulates," Advanced Drug Delivery Reviews, vol. 34, no. 2-3, pp. 135-154, 1998.

[33] G. Cui, L. Wang, P. J. Davis, M. Kara, and H. Liu, "Preparation and physical characterization of a novel marine oil emulsion as a potential new formulation vehicle for lipid soluble drugs," International Journal of Pharmaceutics, vol. 325, no. 1-2, pp. 180$185,2006$.

[34] H. Takeuchi, J. Thongborisute, Y. Matsui, H. Sugihara, H. Yamamoto, and Y. Kawashima, "Novel mucoadhesion tests for polymers and polymer-coated particles to design optimal mucoadhesive drug delivery systems," Advanced Drug Delivery Reviews, vol. 57, no. 11, pp. 1583-1594, 2005.

[35] R. Bhandari and I. P. Kaur, "Pharmacokinetics, tissue distribution and relative bioavailability of isoniazid-solid lipid nanoparticles," International Journal of Pharmaceutics, vol. 441, no. 1-2, pp. 202-212, 2013.

[36] J. Jaiswal, S. K. Gupta, and J. Kreuter, "Preparation of biodegradable cyclosporine nanoparticles by high-pressure emulsification-solvent evaporation process," Journal of Controlled Release, vol. 96, no. 1, pp. 169-178, 2004.

[37] R. H. Müller, K. H. Wallis, S. D. Tröster, and J. Kreuter, "In vitro characterization of poly(methyl-methaerylate) nanoparticles and correlation to their in vivo fate," Journal of Controlled Release, vol. 20, no. 3, pp. 237-246, 1992.

[38] Z. Panagi, A. Beletsi, G. Evangelatos, E. Livaniou, D. S. Ithakissios, and K. Avgoustakis, "Effect of dose on the biodistribution and pharmacokinetics of PLGA and PLGA-mPEG nanoparticles," International Journal of Pharmaceutics, vol. 221, no. 1-2, pp. 143-152, 2001.

[39] G. Mittal, D. K. Sahana, V. Bhardwaj, and M. N. V. Ravi Kumar, "Estradiol loaded PLGA nanoparticles for oral administration: 
effect of polymer molecular weight and copolymer composition on release behavior in vitro and in vivo," Journal of Controlled Release, vol. 119, no. 1, pp. 77-85, 2007.

[40] S. Mao, J. Xu, C. Cai, O. Germershaus, A. Schaper, and T. Kissel, "Effect of WOW process parameters on morphology and burst release of FITC-dextran loaded PLGA microspheres," International Journal of Pharmaceutics, vol. 334, no. 1-2, pp. 137148, 2007. 

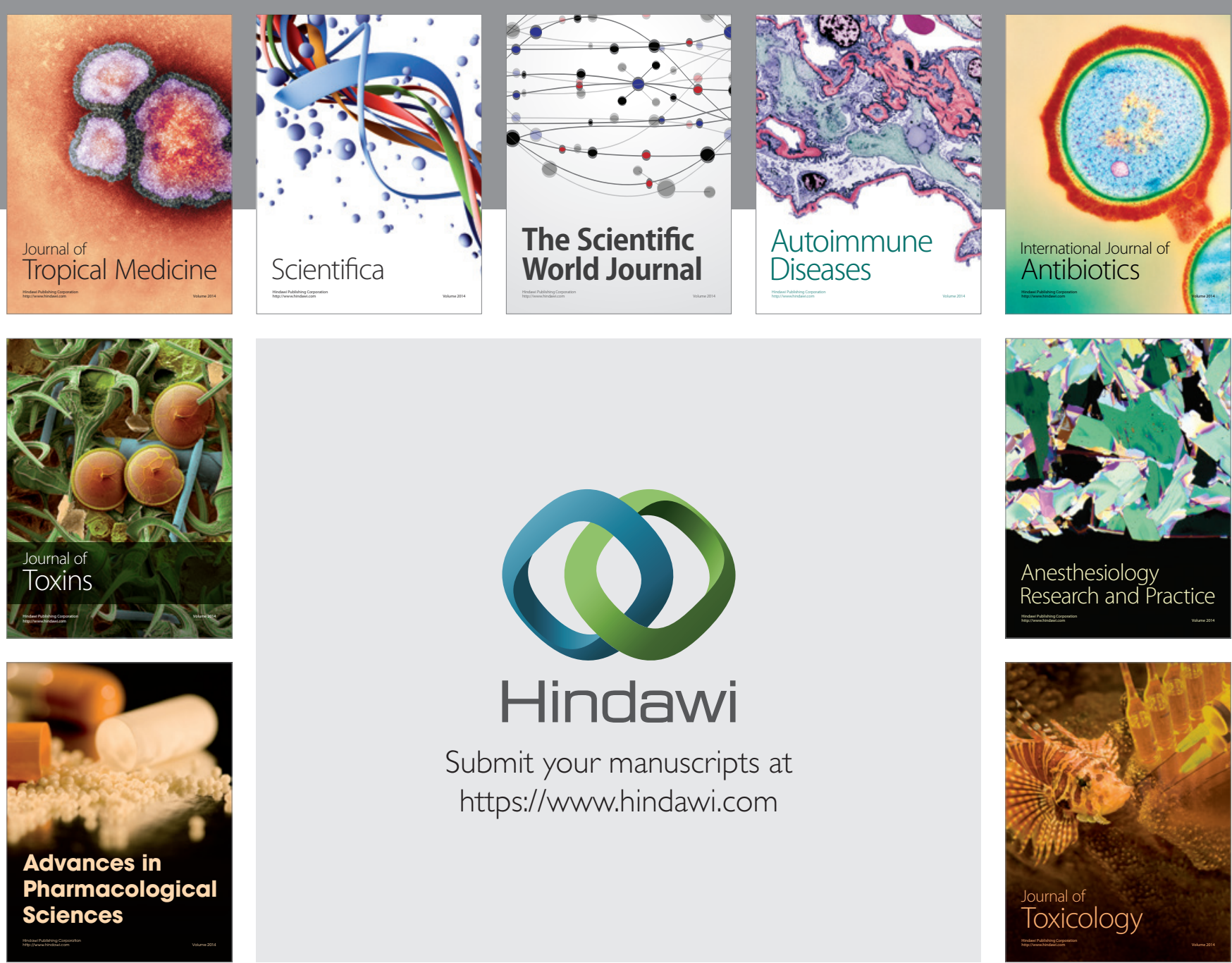

\section{Hindawi}

Submit your manuscripts at

https://www.hindawi.com
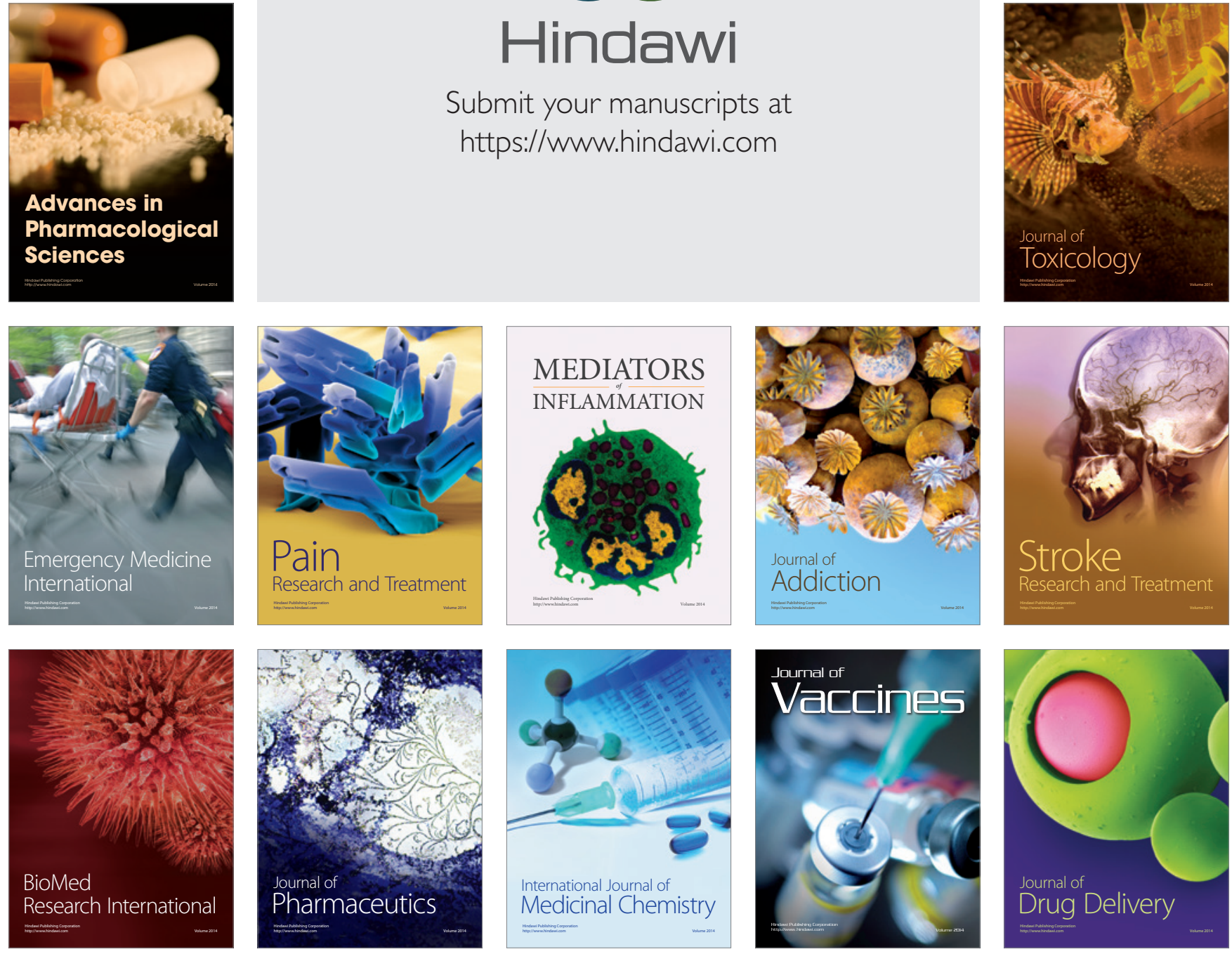